\title{
Satellite based oil spill and ship detection service - from national towards pan-European multi-user services
}

\author{
J. Petter Pedersen, L. Steinbakk, T. Schonberg \& M. Indregard \\ Kongsberg Satellite Services, N-9291 Tromsø, Norway
}

\begin{abstract}
Satellite based Synthetic Aperture Radar (SAR) has become one of the most important sensors for operational monitoring of the marine environment. Kongsberg Satellite Services (KSAT) has since 1994 been delivering SAR based oil spill and ship detection services to European end users in near real time, i.e. less than one hour after satellite overpass. The technical capabilities have been documented and accepted, and the main challenge now is to obtain long-term sustainable services.

The Norwegian SatOcean program and the ESA supported Marked Development project on Marine Surveillance (ESA-MD MAS) are two initiatives where the multi-user model has been established. These have been established to obtain a better understanding and to gain experience relevant for further development of a sustainable pan-European marine surveillance service.

Traditionally KSAT provided a single user in each area with data and information; however it became evident that there was a problem contracting users on a long term basis due mainly to budget restraints. KSAT engaged in a new approach; the multi-user model. In this multi-user model several users share costs, data and information creating a sustainable operational service.
\end{abstract}

Keywords: satellite, SAR, oil service, AIS, multiuser, SatOcean, marine surveillance.

\section{Introduction}

Marine surveillance is important for environmental protection and sustainable management of the Exclusive Economic Zone (EEZ) of coastal nations. National legislation and international agreements exist to prevent illegal activities such as: 
oil pollution, fisheries incursion and smuggling. The majority of European coastal nations have already dedicated marine surveillance programs. These programs are operated by national authorities and are based on a combination of coastal radar, airborne and vessel surveillance systems. During the latter years several European coastal nations have utilized satellite monitoring as an integrated part of their surveillance system.

Satellite-born Synthetic Aperture Radar (SAR) can provide large area coverage of the Earth's surface independent of weather and light conditions. SAR has evolved to become one of the most important sensors for operational monitoring of the marine environment.

Kongsberg Satellite Services (KSAT) in Tromsø, Norway has provided a service utilizing satellite SAR images for detection of oil spill since 1994. The continuing development into an operational service has been conducted in close cooperation with key end-users, starting with the Pollution Control Authority in Norway as the first user. The service chain now meets the most important user requirements. It is also well accepted among the users that the combination of aircraft and satellite is a cost-effective solution for large area surveillance. An increasing interest is also recognized among users, and their financial contribution and commitment has increased year-by-year. But still the service contracts are on a yearly basis and they depend heavily upon the customer's annual surveillance budgets. These uncertainties can reduce the operational value of the service.

The main challenge is therefore to establish a sustainable, operational panEuropean surveillance service. Information and cost sharing models based on harmonized user requirements seem to the preferred strategy. The Norwegian SatOcean[1] program under the responsibility of the Norwegian Space Centre and the ESA supported Marked Development project on Marine Surveillance (ESA-MD MAS) [2] are two initiatives where the multi-user service concept has been established and evaluated. Experience obtained through these projects will form the basis for further development of a pan-European SAR-based oil and ship detection service.

\section{Service chain}

The KSAT oil and ship detection service chain is currently based on SAR data from the European Envisat and the Canadian Radarsat-1 satellites. The service chain (figure 1) includes user dialogue for satellite tasking requests and aircraft operations coordination, acquisition and processing of SAR data, image analysis and interpretation, followed by early warning alert to the customer.

The core elements of the service rely upon the powerful RADARSAT and ENVISAT SAR processing capable of generating a Radarsat ScanSar Image in 7 minutes and an ASAR Wide swath image in $<20$ minutes. It has previously been documented that radar images with a reduced spatial resolution of 100 meters is very suitable for detecting oil on the sea surface $[3,4,5,6]$. 


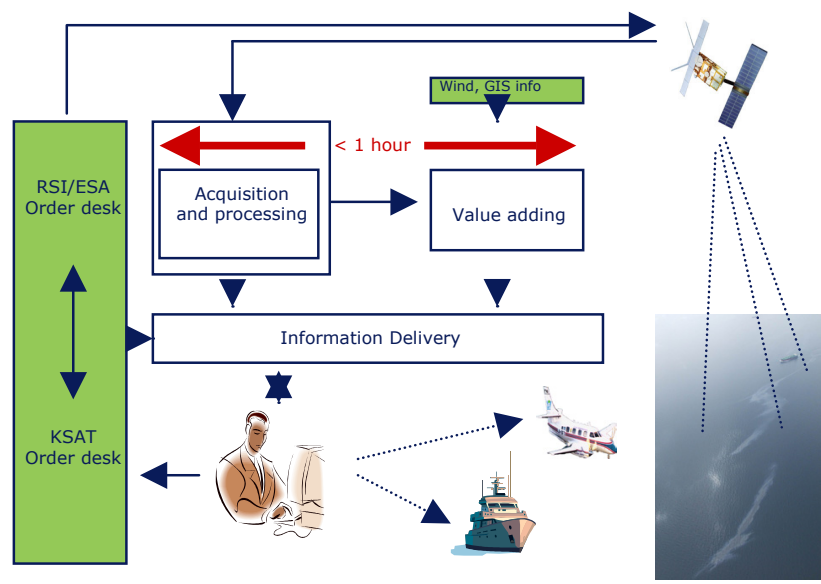

Figure 1: KSAT oil and ship detection service chain.

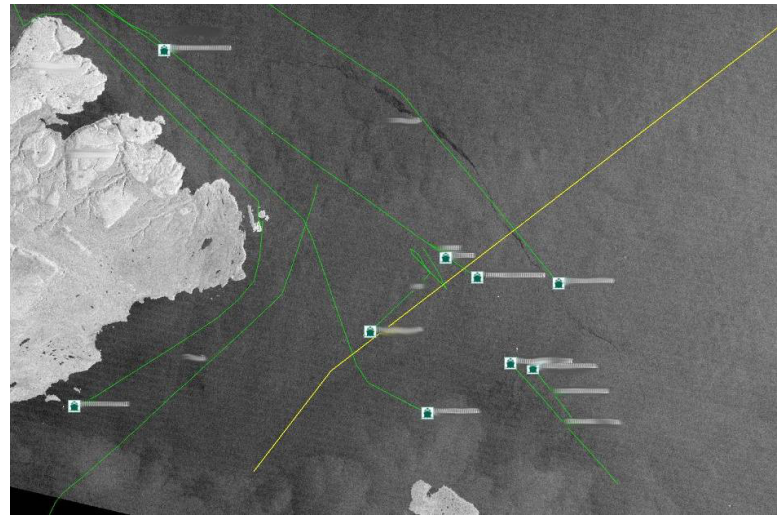

Figure 2: Oil spill in the Barent Sea with AIS information.

The current analysis relies upon interactive human interpretation supported by meteorological and geographical information. KSAT operating engineers have analyzed several thousands of SAR images, and thereof developed their experience and knowledge on interpretation of SAR images in terms of identifying possible oil spills.

A dedicated service workstation developed by Kongsberg Spacetec is applied for information extraction, presentation and distribution. The operator utilizes the integration of SAR data, meteorological data and geographical information during the analysis and interpretation process. To maintain an updated maritime picture for ship traffic monitoring the system is now being upgraded to include information from the Automatic Identification System (AIS) that will be integrated with satellite based ship detection. Ongoing projects from August 2005 have successfully prepared many tests showing the efficiency and accuracy of the integrated information extracts. Figure 2 illustrates KSAT's system with 
integrated AIS information. KSAT expects the upgraded system to be fully operational during Spring 2006.

Customers are informed about possible oil spills within 1 hour after data acquisition. They receive an email report with the results from the analysis. The service information is also made available at the customer's web-page. The web page also contains updated information on planned satellite passes, which is of great importance for coordination with aerial surveillance.

The users have an aircraft stand-by or already flying in the area to check reported oil spills. The delivery time is therefore crucial for this integrated service. The requested delivery time is $<30$ minutes, but $<1$ hour is acceptable.

A user feedback function has also been implemented on the web-page, and the users can now provide KSAT with results (text and images) from verifying flights. This feedback is also essential for KSAT in order to improve service performance.

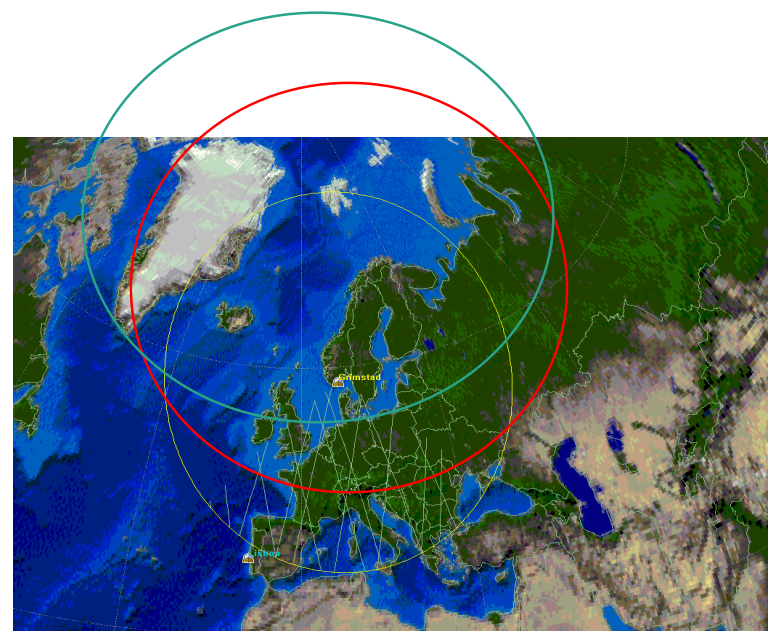

Figure 3: Reception mask of the Svalsat Station (green) Tromsø Station (red) and Grimstad (yellow).

The primary service area includes the coastal waters in Northern Europe within the KSAT direct acquisition area, as illustrated in figure 3.

The capabilities to provide a global coverage service has been documented through the use of the KSAT ground station located at Svalbard at $80^{\circ} \mathrm{N}$ which can access all the 14 orbits of a polar orbiting satellite. The global concept is to downlink on-board recorded data at Svalbard, transmit the raw data via the large bandwidth fiber connection to Tromsø for processing, analysis and information distribution. Tests performed have shown that KSAT is able to deliver ship detection services within 2 hours based on this concept. 


\section{Development of multi-user models}

KSAT experience a growing interest for near-real time oil spill and ship detection services. But still, we have not reached a stage of sustainable service with long-term commitment from paying customers. The current services are delivered on an annual basis and the temporal and geographical coverage are to a large extent determined by the limited surveillance budget for each nation. The customers have not been able to commit to long-term service contracts based on commercial pricing policies.

The service cost is based on a per-image cost-model. The traditional service model has been one service set-up for each customer. This results in the customer paying the full cost of the service, in which they don't utilize the full potential of the satellite. In addition, no coordination between customers has existed previously, therefore frequent acquisitions-request conflicts have occurred.

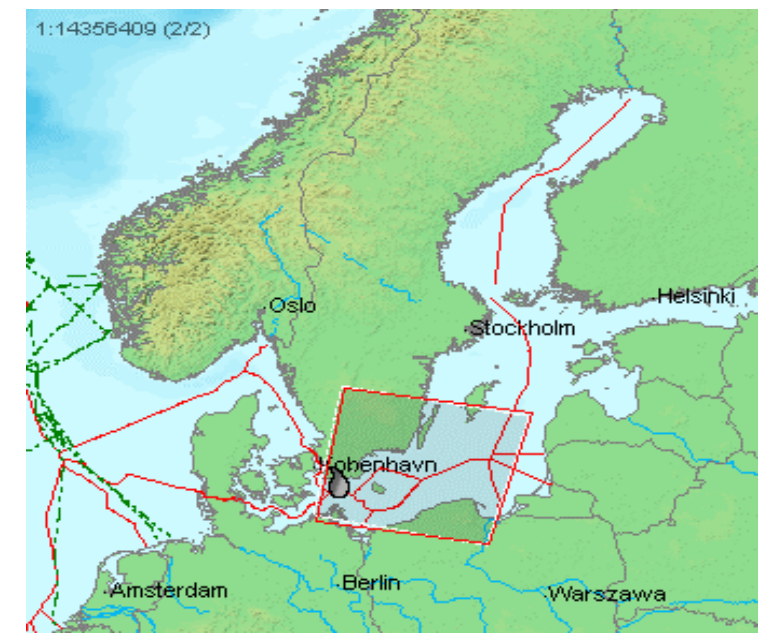

Figure 4: $\quad$ Illustration of ASAR Wide Swath coverage.

As seen from figure 4 above the wide swath modes covers a wide area of the sea and for some areas an image often covers more than one EEZ.

In the Baltic a single ASAR Wide Swath image can cover fractions of up to 6 nations EEZ. The existing cost model might be acceptable for nations with a considerable EEZ, but small coastal nations must pay the total cost of the service, even though only a small part of the image covers their EEZ. This makes the service less accessible for nations with a small EEZ.

There has been little coordination among users of EO-based services, in spite the fact that oil spills often affects neighboring countries as well. With the traditional single user model an identified possible oil spill was only reported to the buying customer, even if the spill was identified in another nation's EEZ. 
This model was therefore a blockage for the development of a sustainable panEuropean service.

To improve, an initiative to develop multinational services has been taken through the Marine Surveillance (MAS) Market Development Project. ESA's Marked Development Program supports the initiative. Through this project, KSAT initiated a close cooperation with key member states in the Baltic and the North Sea. The idea was to utilize the large coverage of ASAR to develop a harmonized information and cost-sharing service for the two areas. Establishing a single harmonized service for each area could be the first step towards a sustainable service.

The first operational trial started in March 2004 in cooperation with Maritime and Coastguard Agency (UK), The North Sea Directorate (NL) and Federal Institute of Hydrology (DE) as paying customers. In October Belgium, represented by Management Unit of the North Sea Mathematical Models (MUMM) joined the service. The service cost for each nation is calculated based on temporal and geographical coverage of the nations EEZ, and is therefore a proportion of the size of the EEZ. With this model the countries pay only for the part of the satellite image that actually covers their area. This represents a more correct costing of the service and for small coastal nations this service model represents an opportunity to start using satellite surveillance.

A slightly different model has been tested in the Baltic where Finland and Sweden coordinates their satellite orders. All requested images are included in one joint service and all countries participating receive results from the analysis performed on these images, e.g. the result of an oil spill analysis from an image ordered and paid for by Sweden will also be distributed to Finland. Poland has recently been included in the service for a limited trial period. The countries also coordinate flight surveillance for images covering more than one EEZ, and the three countries agree in advance who is responsible for surveillance in the area. Diplomatic clearance has been agreed for flying into each other's area. This approach results in a more cost-effective utilization of the aircrafts and an improved surveillance for the whole area.

These multi national services have been successful and the feedback from the users has been very positive. This new model contributes to a significant cost reduction for each country and it also improves the cooperation between neighboring countries. For the North Sea service the cost for each nation has decreased and the number of satellite images used to monitor the area has increased significantly compared to the period with single user services in the area.

Today KSAT delivers a single user service to Denmark and Norway, and they have both expressed interest in participating in the multinational service.

SatOcean is a national multi-user program managed by the Norwegian Space Center (NSC). The aim of this program is to establish a cost-effective use of satellite data for the purpose of national surveillance and monitoring. KSAT is the responsible service provider under the responsibility of NSC. The program activities include documentation of the national public user needs for access to surveillance and monitoring information in particular over the large ocean areas, 
pilot service deliveries involving the national users responsible for oil spill detection, ship traffic detection and ice mapping and monitoring. Another important activity is to aggregate the user requirements into a harmonized multiuser model that will be applied for requesting access to the satellite SAR data from the satellite operators and owners.

KSAT is now merging all the existing services into one single North European oil spill service where all nations involved will share one service.

The main benefits for each participating nation are lower costs, more satellite coverage and improved access to information of all oil spill pollutions reported. It also represents a basis for joint flight surveillance planning. The feedback function where the customer reports verifications of checked oil spills is also available for all service member states and can be used to inform neighboring countries about the status of a reported spill (confirmed, not confirmed) and the action taken.

It is, however, expected that large efforts will be needed to become fully successful with this model. A stepwise approach has therefore been applied, using the most experienced users as brokers versus new users.

Integration of AIS ship identification could increase the value of the satellite service substantially and would lead to a more effective use of other complementary resources such as surveillance vessels.

\section{Conclusions and recommendations}

One of the most important blockages for further development of the oil spill and ship detection services have been the lack of international framework and coordination among end-users. This combined with dependency of individual nation's annual surveillance budget and high service costs have been among the main bottlenecks for sustainable service development.

These trials have documented that the cost -benefit of the multinational oil spill service has improved compared to the traditional single user services. However, the main challenge is still to achieve long-term commitment from the end users. An important requirement is that such a service must provide comparable information and quality for all European waters. With such a service model established throughout Europe service costs and information can be shared among regional, national and international authorities. All contributing and committed to collaborate to achieve a cost effective, coordinated and sustainable surveillance of the European EEZ.

The technical and operational concept have been demonstrated and will be expanded to one single service available for paying customers in the Baltic, Barents Sea, Norwegian Sea, North Sea and the English Channel.

To achieve a pan-European service available for all coastal nations, future European activities should build on experience obtained through these trials. The main focus should be to include costal nations with no satellite surveillance budget. The core challenge in the future is programmatic rather than technical, i.e. to define the roles and responsibilities of the individual nations compared to Pan-European entities. 


\section{References}

[1] J.P. Pedersen, Sathav program, KSAT report 2004.

[2] L. Steinbakk, JP Pedersen, M. Indregard, ESA/EOMD of Envisat Marine Surveillance Services- SAR based oil and shiop detection services. ERS/Envisat Symposium, Salzburg, Austria, September 2004.

[3] J.P. Pedersen, T. Bauna, .G. Seljelv, F. Landmark, J.H. Andersen, Earth Observation - a Cost-effective Improvement for Oil Spill Monitoring, Proceedings of the 27. ISPRS conference, Tromsø, Norway, 1998.

[4] J.P. Pedersen, F. Landmark, T. Bauna, R.T. Enoksen, Oil Spill Detection Activities in Norwegian Waters, TSS Report TSS-OLJ-1627/JPP-96, 11.11.96, Paper presented at RADARSAT Workshop 19-21.11.96.

[5] T. Wahl, Å. Skøelv, T. Anderssen, J.P. Pedersen, J.H. Andersen, O.A. Follum, G.D. Strøm, T-I. Bern, H. Hamnes, R. Solberg, Radar satellites: A new tool for pollution monitoring in coastal waters, Coastal Management 24:61-71, 1996.

[6] J.P. Pedersen (Editor), Utilisation of ERS SAR Data for Near real-time Detection of Oil Spills at Sea, TSS Report TSS-CEO-008/JPP-96, February 1996, Executive Summary from Final Report to the EU/CEO Applications Proof-of-Concept Study.

[7] P. Pedersen, L.G. Seljelv, T. Bauna, G.D. Strøm, O.A. Follum, J.H. Andersen, T. Wahl, Å. Skøelv, Towards an Operational Oil Spill Detection Service in the Mediterranean ? The Norwegian Experience: A Preoperational Early Warning Detection Service Using ERS SAR Data, Spill Science \& Technology Bulletin, Vol. 3, No 1/2, pp. 41-46, 1996. 